\title{
Processing and Characterization of Bulk FeSe
}

\author{
Miryala Muralidhar ${ }^{1}$, Tomokazu Hanai ${ }^{1}$, Kouichi Furutani ${ }^{1}$, Pavel Diko ${ }^{2}$, Milos Jirsa ${ }^{3}$ and Masato Murakami ${ }^{1}$ \\ 1. SRL, Graduate School of Science and Engineering, Shibaura Institute of Technology, Tokyo 135-8548, Japan \\ 2. Institute of Experimental Physics SAS, Watsonova 49, SK-04353 Kosice, Slovak Republic \\ 3. Institute of Physics CAS, Na Slovance 2, CZ-18221 Praha 8, Czech Republic
}

\begin{abstract}
We investigated the effect of processing conditions on the structure and physical properties of the polycrystalline samples of $\mathrm{Ca}_{0.8} \mathrm{Fe}_{2} \mathrm{Se}_{2}$ prepared via solid state reaction. X-ray diffraction showed that the main phase became FeSe when the sintering temperature increased above $350{ }^{\circ} \mathrm{C}$. The temperature dependence of magnetization ( $M-T$ curves) recorded in zero-field-cooled mode by SQUID magnetometer showed some bumps at around $120 \mathrm{~K}$ to $140 \mathrm{~K}$. These bumps moved to lower temperatures when the processing temperature increased above $550{ }^{\circ} \mathrm{C}$. On the other hand, pure FeSe materials produced at $900{ }^{\circ} \mathrm{C}$ for $24 \mathrm{~h}$ showed a sharp superconducting transition $T_{c, \text { onset }}=8.16 \mathrm{~K}$ and the critical current density of $6,252 \mathrm{~A} / \mathrm{cm}^{2}$ at $5 \mathrm{~K}$, self-field. SEM results indicated an enhanced grain connectivity.
\end{abstract}

Key words: $\mathrm{Ca}_{0.8} \mathrm{Fe}_{2} \mathrm{Se}_{2}$, FeSe, X-ray diffraction, magnetization measurements, SEM, EDX analysis.

\section{Introduction}

The discovery of superconductivity in Fe-based quaternary oxypnictide $\mathrm{LaFeAsF}_{x} \mathrm{O}_{1-x}$ with the transition temperature $T_{c} \approx 26 \mathrm{~K}$ [1] has motivated researchers in the endeavor to improve performance of this compound for practical applications. The substitution of La by smaller RE (rare earth) elements led to up to now the highest transition temperature in this class of compounds, $T_{c} \approx 56 \mathrm{~K}[2,3]$. Researchers have until now identified eleven systems of pnictides. Among them, the greatest interest has been devoted to FeSe, $\mathrm{FeTe}_{1-x} \mathrm{Se}_{x}$, and $\mathrm{FeTe}_{1-x} \mathrm{~S}_{x}$, due to their simple crystal structure and absence of the toxic As [4]. The onset $T_{C}$ of $15 \mathrm{~K}$ was found in $\mathrm{FeSe}_{0.5} \mathrm{Te}_{0.5}$ [5-7], while in the bulk $\mathrm{K}_{0.8} \mathrm{Fe}_{2} \mathrm{Se}_{2} T_{\mathrm{c}}$ was $31 \mathrm{~K}$ and in the single crystal of $\mathrm{Cs}_{0.8} \mathrm{Fe}_{2} \mathrm{Se}_{2} T_{\mathrm{c}}$ reached $27.4 \mathrm{~K}$ [8]. Note that besides the very promising values of the upper critical field, comparable to those of the cuprate RE-123 superconductors, the iron-pnictide materials offer several advantages in the preparation as compared to the cuprate high- $T_{\mathrm{c}}$ superconductors: no oxygenation

Corresponding author: Miryala Muralidhar, Prof., research fields: high $T_{c}$ superconducting materials. E-mail: miryala1@shibaura-it.ac.jp. process is required, "clean" chemistry can be used to obtain large and homogeneous samples. There is no twinning complicating interpretation of the magnetization data $[9,10]$. This makes the pnictides very interesting for applications, where large and homogenous samples are needed. Among the As-free pnictide superconductors the recently reported $\mathrm{X}_{\mathrm{y}} \mathrm{Fe}_{2} \mathrm{Se}_{2}$ ones, with $\mathrm{X}=\mathrm{Yb}, \mathrm{Li}, \mathrm{Ca}, \mathrm{Na}, \mathrm{Eu}$, Ba etc., belong to the most promising materials. The system offers high $T_{\mathrm{c}}$ and $H_{\mathrm{c} 2}$ values and a simple chemistry [11]. E.g., in the CaFeSe system, $T_{c}$ as high as $40 \mathrm{~K}$ was reported. For applications, a material with high super-currents and a reproducible performance is required. In order to improve the critical current density of this material, we studied the processing conditions.

In the present work, high quality polycrystalline samples of $\mathrm{Ca}_{0.8} \mathrm{Fe}_{2} \mathrm{Se}_{2}$ were prepared and the effect of $\mathrm{Ca}$ addition on microstructure, and magnetic performance was investigated. There is a large difference between the melting point of $\mathrm{Fe}$ and Se. We prepared the first batch of the $\mathrm{Ca}_{0.8} \mathrm{Fe}_{2} \mathrm{Se}_{2}$ samples at various, rather low sintering temperatures. Eventually, the same material was produced at a high temperature. 
For comparison, pure FeSe material was produced by utilizing the $6 \mathrm{~h}$ ball milled powders processed at $900{ }^{\circ} \mathrm{C}$ for $24 \mathrm{~h}$. All samples were measured by the $M-T$ measurements. Further, SEM (scanning electron microscopy) and SEM by EDX (energy dispersive $\mathrm{X}$-ray) analyses were performed.

\section{Experimental}

The $\mathrm{Ca}_{0.8} \mathrm{Fe}_{2} \mathrm{Se}_{2}$ material was produced from the commercial powders of $\mathrm{Fe}$ ( $99.9 \%$ purity), Se (99.5\% purity) and $\mathrm{CaO}$ (99\% purity). The powders were mixed in a nominal ratio $\mathrm{Ca}: \mathrm{Fe}: \mathrm{Se}=0.8: 2: 2$ in a glove box and thoroughly ground there in argon atmosphere. The powder mixture was then pressed into pellets 5 $\mathrm{mm}$ in diameter and $2 \mathrm{~mm}$ thick using a uniaxial pressure of $200 \mathrm{MPa}$. The consolidated pellets were wrapped into $\mathrm{Nb}$ foil, then sintered in two steps. In the first step, utilizing a quartz tube furnace, the samples were sintered in a high purity $\mathrm{Ar}$ gas at $250{ }^{\circ} \mathrm{C}$ for 10 hours. Then, the sintered pellets were once more thoroughly ground and mixed in a glove box and again pressed into the pellets similar to the first step. In the second step, the samples were placed in an alumina crucible in a quartz tube furnace and there kept in a high purity argon atmosphere for $10 \mathrm{~h}$ at different sintering temperatures from $250{ }^{\circ} \mathrm{C}$ to $550{ }^{\circ} \mathrm{C}$ with increment of $50{ }^{\circ} \mathrm{C}$. Eventually, the furnace was cooled to room temperature. Some of the samples were sealed in quartz tubes and sintered at high temperatures at $700{ }^{\circ} \mathrm{C}$ for $10 \mathrm{~h}$, and at $900{ }^{\circ} \mathrm{C}$ for $15 \mathrm{~h}$. Finally, the temperature was lowered to room temperature at a cooling rate of $50{ }^{\circ} \mathrm{C} / \mathrm{h}$. For the pure FeSe material was produced.

The constituent phases of the samples were identified with a high-resolution automated X-ray powder diffractometer (RINT2200), using $\mathrm{Cu}-\mathrm{K}_{\alpha}$ radiation generated at $40 \mathrm{kV}$ and $40 \mathrm{~mA}$. Small specimens with dimensions of $1 \times 1 \times 0.5 \mathrm{~mm}^{3}$ were cut from the bulk FeCaSe pellets and subjected to the measurements of magnetization vs. temperature, using a SQUID magnetometer (Quantum Design, model
MPMS5) in the temperature range $5 \mathrm{~K}$ to $300 \mathrm{~K}$. The microstructure of the samples sintered at high temperatures was studied using the SEM and the chemical composition of various phases was analyzed by EDX spectroscopy.

\section{Results and Discussion}

Fig. 1 presents the X-ray diffraction patterns of the $\mathrm{Ca}_{0.8} \mathrm{Fe}_{2} \mathrm{Se}_{2}$ compounds produced at different low temperatures. In the samples sintered below $350{ }^{\circ} \mathrm{C}$ the dominant phases were $\mathrm{FeSe}, \mathrm{Ca}_{4} \mathrm{Fe}_{9} \mathrm{O}_{17}$ and $\mathrm{SeO}_{2}$. With increasing temperature the peak of $\mathrm{SeO}_{2}$ disappeared and the main phase became $\beta$-FeSe, with a small minority of $\mathrm{Ca}_{4} \mathrm{Fe}_{9} \mathrm{O}_{17}$. X-ray diffraction indicated that $\mathrm{FeSe}$ samples were polycrystalline, similar to the earlier reports $[1,12]$. The main $\mathrm{Ca}_{4} \mathrm{Fe}_{9} \mathrm{O}_{17}$ peak decreased with increasing temperature, indicating that $\mathrm{Ca}$ could enter the Fe sublattice site of FeSe. More details will be discussed further.

The temperature dependence of the $\mathrm{dc}$ magnetization curves of $\mathrm{Ca}_{0.8} \mathrm{Fe}_{2} \mathrm{Se}_{2}$ recorded in $\mathrm{ZFC}$ (zero-field-cooled) mode showed a significant bump at $133 \mathrm{~K}$ to $135 \mathrm{~K}$. This high temperature bump shifted to lower temperatures with the sintering temperature increasing above $500{ }^{\circ} \mathrm{C}$, and reached 123 $\mathrm{K}$ for the sintering temperature of $750{ }^{\circ} \mathrm{C}$ (see Fig. 2). Similar bumps were observed by Yoshikazu et al. in

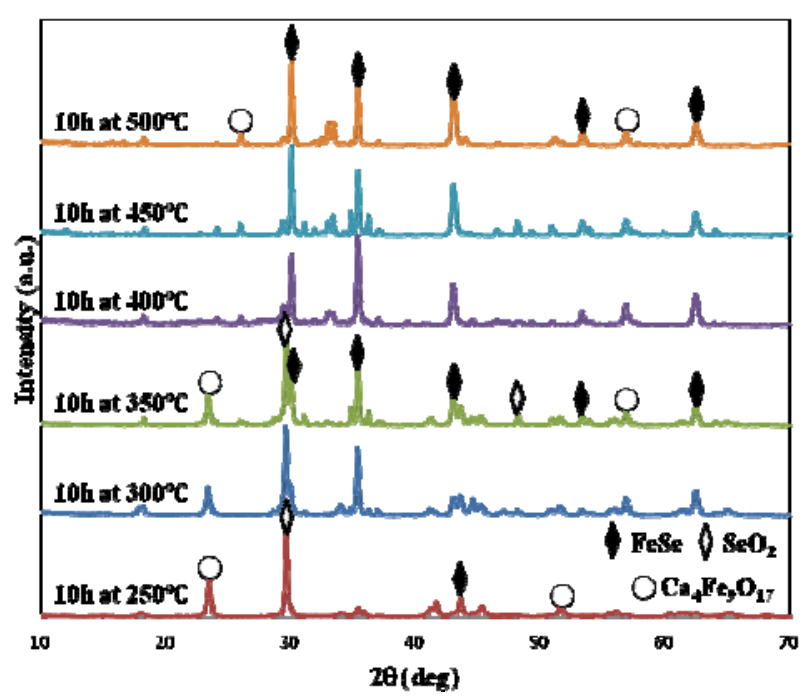

Fig. 1 X-ray diffraction patterns of $\mathrm{Ca}_{0.8} \mathrm{Fe}_{2} \mathrm{Se}_{2}$ material. 
the FeSe material under high pressure at around 50 and $200 \mathrm{~K}$, and attributed them to the structural phase transition and the magnetic transition, respectively [13]. According to Li et al. [14], a similar anomaly near $125 \mathrm{~K}$ was observed also in sintered bulk $\mathrm{FeSeCr}_{0.05}$ materials in magnetization versus temperature dependence near $125 \mathrm{~K}$, in both the zero-field-cooled and field cooled (20 Oe) modes.

The micro structure and chemical composition of the newly prepared $\mathrm{Ca}_{0.8} \mathrm{Fe}_{2} \mathrm{Se}_{2}$ material were studied by means of SEM and SEM by EDX analyses.

The morphology and chemical composition of $\mathrm{Ca}_{0.8} \mathrm{Fe}_{2} \mathrm{Se}_{2}$ samples sintered at high temperatures, $750^{\circ} \mathrm{C}$ and $900{ }^{\circ} \mathrm{C}$, were carried out utilizing the SEM, SEM by EDX analyses. The typical scanning electron micrographs for the polished sample surface of as grown $\mathrm{Ca}_{0.8} \mathrm{Fe}_{2} \mathrm{Se}_{2}$ material treated for 10 hours at
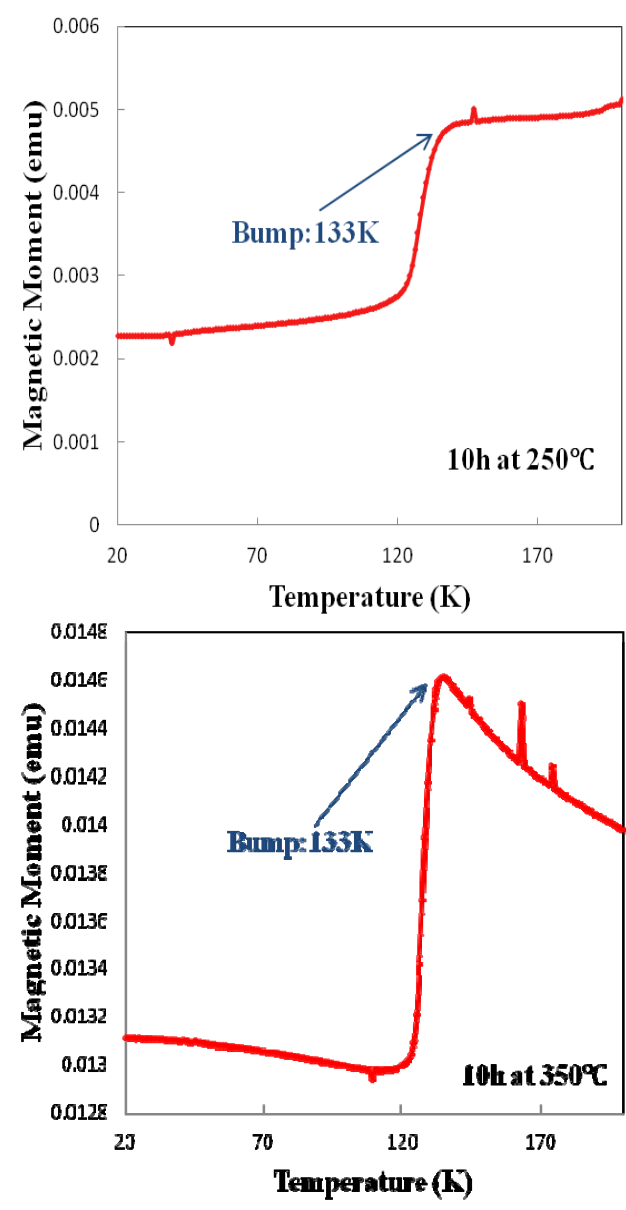

Fig. 2 Magnetization versus temperature curves for the $\mathrm{Ca}_{0.8} \mathrm{Fe}_{2} \mathrm{Se}_{2}$ samples sintered in argon atmosphere at $250{ }^{\circ} \mathrm{C}, 350{ }^{\circ} \mathrm{C}$, $450{ }^{\circ} \mathrm{C}$, and $750{ }^{\circ} \mathrm{C}$ for 10 hours.

$750{ }^{\circ} \mathrm{C}$ were depicted in Fig. 3. According to the SEM images and element mapping, the sample contained only a few distinct structures distinguished by 3 colors, black, gray and white (see Fig. 3). However, the SEM by EDX analysis showed many structures (Table 1, see spectra A to I). It indicated that we have not yet reached a homogenous FeSe or FeSeCa compound (see Table 1). On the sample sintered for $15 \mathrm{~h}$ at $900{ }^{\circ} \mathrm{C}$ the SEM micrographs are similar to those of the samples sintered at $750{ }^{\circ} \mathrm{C}$ (see Fig. 4). Further, the elemental mapping exhibits only a few structures containing mainly iron, selenium and calcium (Table 2, spectra a-e).

Note that in the samples sintered for 15 hours at high temperature, around $900^{\circ} \mathrm{C}$, the major phase was FeSe (see Table 2). Based on the experience with FeSe materials produced by low and high temperature
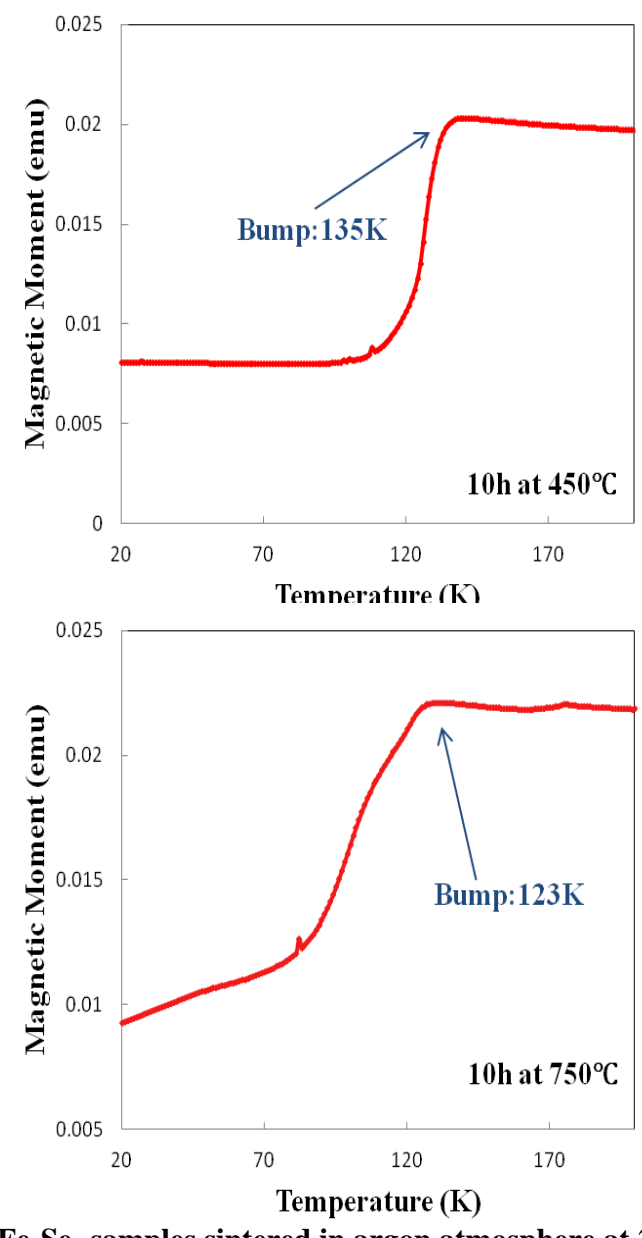


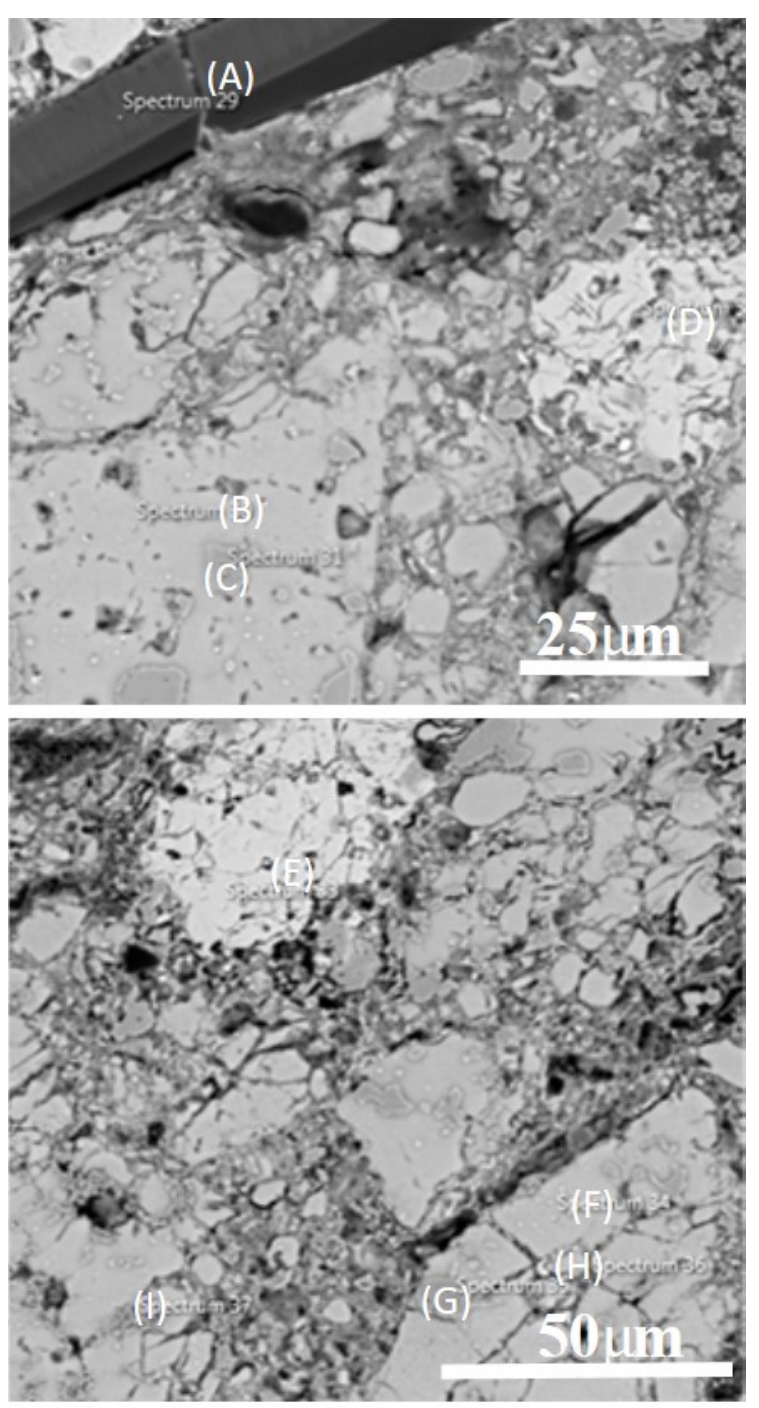

Fig. 3 The SEM images of the $\mathrm{Ca}_{0.8} \mathrm{Fe}_{2} \mathrm{Se}_{2}$ samples sintered for $10 \mathrm{~h}$ at $750{ }^{\circ} \mathrm{C}$.

sintering, one can expect that the high-temperature processing is crucial for growing good quality $\mathrm{FeSe}$ material with high density. Until now, several researchers have tried to produce the FeSe material at around $750{ }^{\circ} \mathrm{C}$. However, they found rather low critical current densities, in the range of $10^{3} \mathrm{~A} / \mathrm{cm}^{2}$ at $5 \mathrm{~K}$, the samples becoming normal even at external magnetic field around $0.4 \mathrm{~T}$ [15]. In the attempt to improve properties of these materials, we mixed the commercial powders of $\mathrm{Fe}(99.9 \%$ purity) and $\mathrm{Se}$ (99.9\% purity) in the nominal ratio $\mathrm{Fe}: \mathrm{Se}=1: 1$, thoroughly ground and ball-milled them in glove box for $6 \mathrm{~h}$. The powder mixture was pressed into pellets of $5 \mathrm{~mm}$ in diameter and $2 \mathrm{~mm}$ thickness using a uniaxial pressure of $200 \mathrm{MPa}$. The samples were sealed in quartz tubes and sintered at $600^{\circ} \mathrm{C}$ for $24 \mathrm{~h}$. Then, the pellets were again thoroughly ground and ball-milled in a glove box and pressed into pellets and eventually final sintering was performed at $900{ }^{\circ} \mathrm{C}$ for $24 \mathrm{~h}$. The phase purity and the superconducting performance were characterized by $\mathrm{x}$-ray diffractometer and SQUID, respectively.

The phase composition of the final bulk FeSe material sintered at $900{ }^{\circ} \mathrm{C}$ for $24 \mathrm{~h}$ is shown in Fig. 5 (top). For comparison, we also show the structure of the $\mathrm{Ca}_{0.8} \mathrm{Fe}_{2} \mathrm{Se}_{2}$ sample sintered at $750{ }^{\circ} \mathrm{C}$ for $10 \mathrm{~h}$. The FeSe material sintered at $900{ }^{\circ} \mathrm{C}$ for $24 \mathrm{~h}$ showed mainly the $\beta$-FeSe phase with tetragonal structure (Fig. 5, top) with a minor amount of impurity of the $\mathrm{Ca}_{0.8} \mathrm{Fe}_{2} \mathrm{Se}_{2}$ sample contains several phases and only isolated FeSe islands as indicated by SEM by EDX analysis (see Table 1). The X-ray diffraction proved that with an optimum sintering process combined with ball-milling FeSe phase formation appears also in the $\mathrm{Ca}_{0.8} \mathrm{Fe}_{2} \mathrm{Se}_{2}$ samples.

Table 1 The SEM by EDX analysis of $\mathrm{Ca}_{0.8} \mathrm{Fe}_{2} \mathrm{Se}_{2}$ sample sintered $10 \mathrm{~h}$ at $750{ }^{\circ} \mathrm{C}$.

\begin{tabular}{lllllll}
\hline & $\mathrm{Fe}(\%)$ & $\mathrm{Se}(\%)$ & $\mathrm{Ca}(\%)$ & $\mathrm{O}(\%)$ & Others & Color \\
\hline (A) & 0.1 & 0.1 & 21.9 & 55.1 & 22.9 & Black \\
(B) & 1.2 & 47.9 & 47.7 & 0 & 3.3 & White \\
(C) & 94.2 & 0.2 & 0.7 & 0 & 4.9 & Gray \\
(D) & 41.1 & 44.2 & 1 & 11.9 & 1.8 & White \\
(E) & 46.9 & 51.8 & 0 & 0 & 1.3 & White \\
(F) & 1.9 & 44.6 & 43.2 & 7.8 & 2.4 & White \\
(G) & 96.2 & 0.2 & 0.8 & 0 & 2.8 & Gray \\
(H) & 1.3 & 30.4 & 23.9 & 40.9 & 3.5 & Gray \\
(I) & 71.6 & 3.3 & 5.1 & 15.5 & 4.5 & Gray \\
\hline
\end{tabular}


Table 2 The SEM by EDX analysis of $\mathrm{Ca}_{0.8} \mathrm{Fe}_{2} \mathrm{Se}_{2}$ sample sintered $15 \mathrm{~h}$ at $900{ }^{\circ} \mathrm{C}$.

\begin{tabular}{llllll}
\hline & $\mathrm{Fe}(\%)$ & $\mathrm{Se}(\%)$ & $\mathrm{Ca}(\%)$ & $\mathrm{O}(\%)$ & Color \\
\hline (a) & 46.9 & 51.8 & 0 & 0 & White \\
(b) & 1.9 & 44.6 & 43.2 & 7.8 & White \\
(c) & 96.2 & 0.2 & 0.8 & 0 & Gray \\
(d) & 1.3 & 30.4 & 23.9 & 40.9 & Black \\
(e) & 71.6 & 3.3 & 5.1 & 15.5 & Gray \\
\hline
\end{tabular}

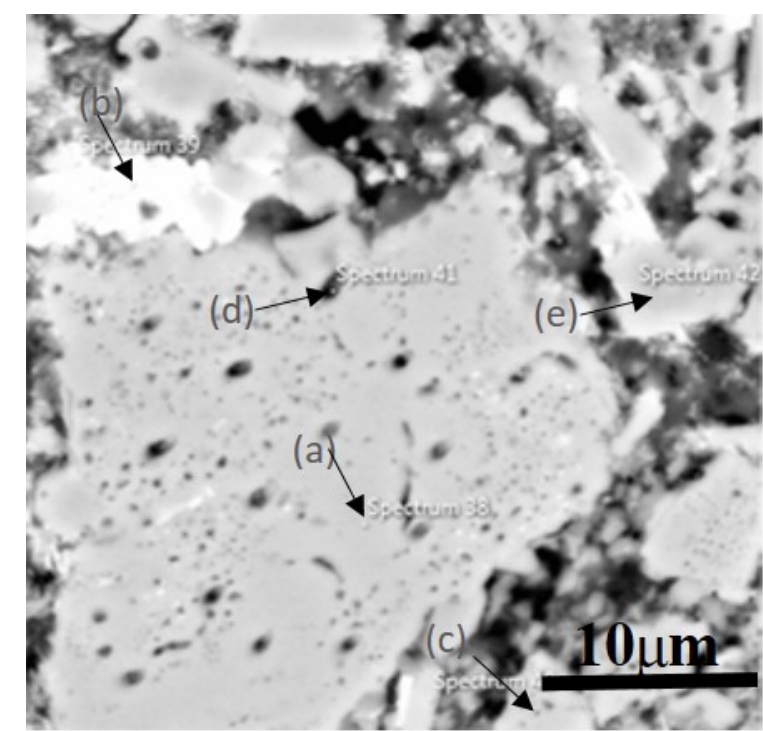

Fig. 4 The SEM images of $\mathrm{Ca}_{0.8} \mathrm{Fe}_{2} \mathrm{Se}_{2}$ samples sintered for $15 \mathrm{~h}$ at $900{ }^{\circ} \mathrm{C}$.

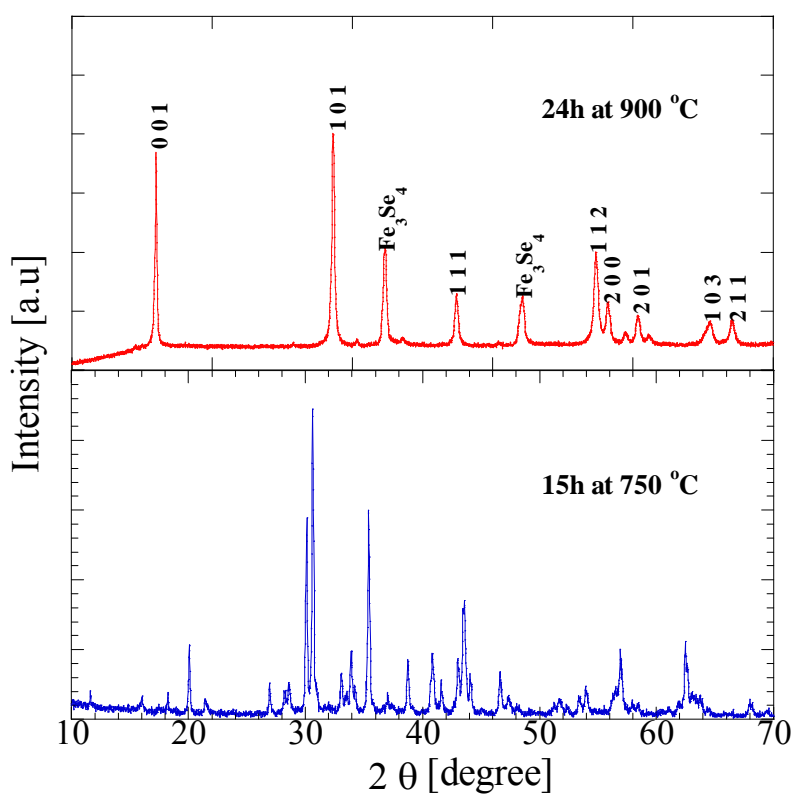

Fig. 5 X-ray diffraction spectra of bulk FeSe sample sintered at $900{ }^{\circ} \mathrm{C}$ for $24 \mathrm{~h}$ (top) and $750{ }^{\circ} \mathrm{C}$ for $15 \mathrm{~h}$ (bottom).

Fig. 6 (left) shows the temperature dependence of the magnetic susceptibility of FeSe sample sintered at
$900{ }^{\circ} \mathrm{C}$ for $24 \mathrm{~h}$. The superconducting transition temperature was $8.16 \mathrm{~K}$. No high temperature bumps were observed. The M-H loop was measured at $5 \mathrm{~K}$ on the same sample and the critical current density was estimated from it using Bean model (Fig. 6, right). The critical current densities $\left(J_{\mathrm{c}}\right)$ recorded at $5 \mathrm{~K}$ at self-field and $2 \mathrm{~T}$ were $6,253 \mathrm{~A} / \mathrm{cm}^{2}$ and 2,597 A/ $\mathrm{cm}^{2}$, respectively. The present $J_{\mathrm{c}}$ and $H_{\text {irr }}$ values are clearly improved as compared to the bulk FeSe materials previously produced by the sintering process $[15,16]$. Further, the low field $J_{c}$ values were also good as compared to the recently reported FeSe wire prepared by diffusion process [16]. The critical current densities of $350 \mathrm{~A} / \mathrm{cm}^{2}$ and $1,027 \mathrm{~A} / \mathrm{cm}^{2}$ were reported for FeSe tapes and several-core FeSe wires with $\mathrm{Fe}$ tapes, respectively [17].

The improved $J_{\mathrm{c}}$ results can originate from a better grain connectivity due to the higher sintering temperature used in the present synthesis process. The results indicate a possibility to further improve the process by a synthesis at high temperatures. To obtain more information on the $\mathrm{FeSe}$ material composition, we studied the microstructure and chemical composition by SEM and SEM-EDX analyses (Fig. 7).

Fig. 7 shows the scanning electron micrograph images of the polished surface of the FeSe material sintered at $900{ }^{\circ} \mathrm{C}$ for $24 \mathrm{~h}$. On the top figure, a high density of FeSe grains can be seen. The SEM-EDX analysis clarified that all white grains were of FeSe. The average grain size of 1-3 micrometers, similar to earlier reports [15], was observed. In a higher magnification (the bottom figure) one can see also small grains of some secondary impurity phases at grain boundaries, marked by black arrows. 

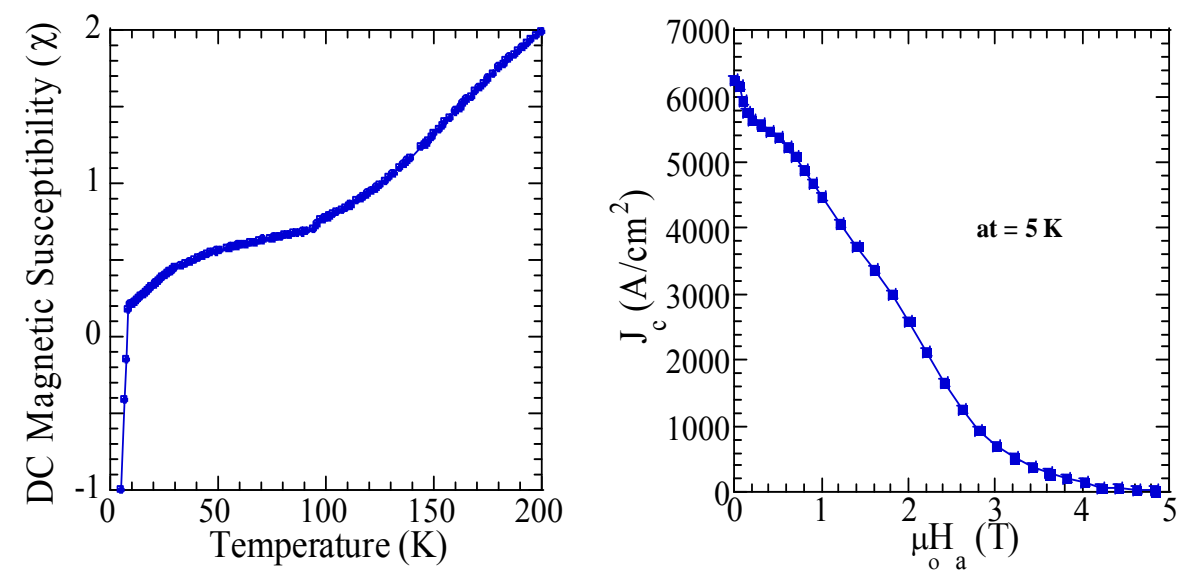

Fig. 6 Temperature dependence of DC magnetic susceptibility for FeSe samples produced by sintering at $900{ }^{\circ} \mathrm{C}$ for 24 hours and ball milling time $6 \mathrm{~h}$ (left) and the field dependence of the critical current density (right) for the same material at 5 K.

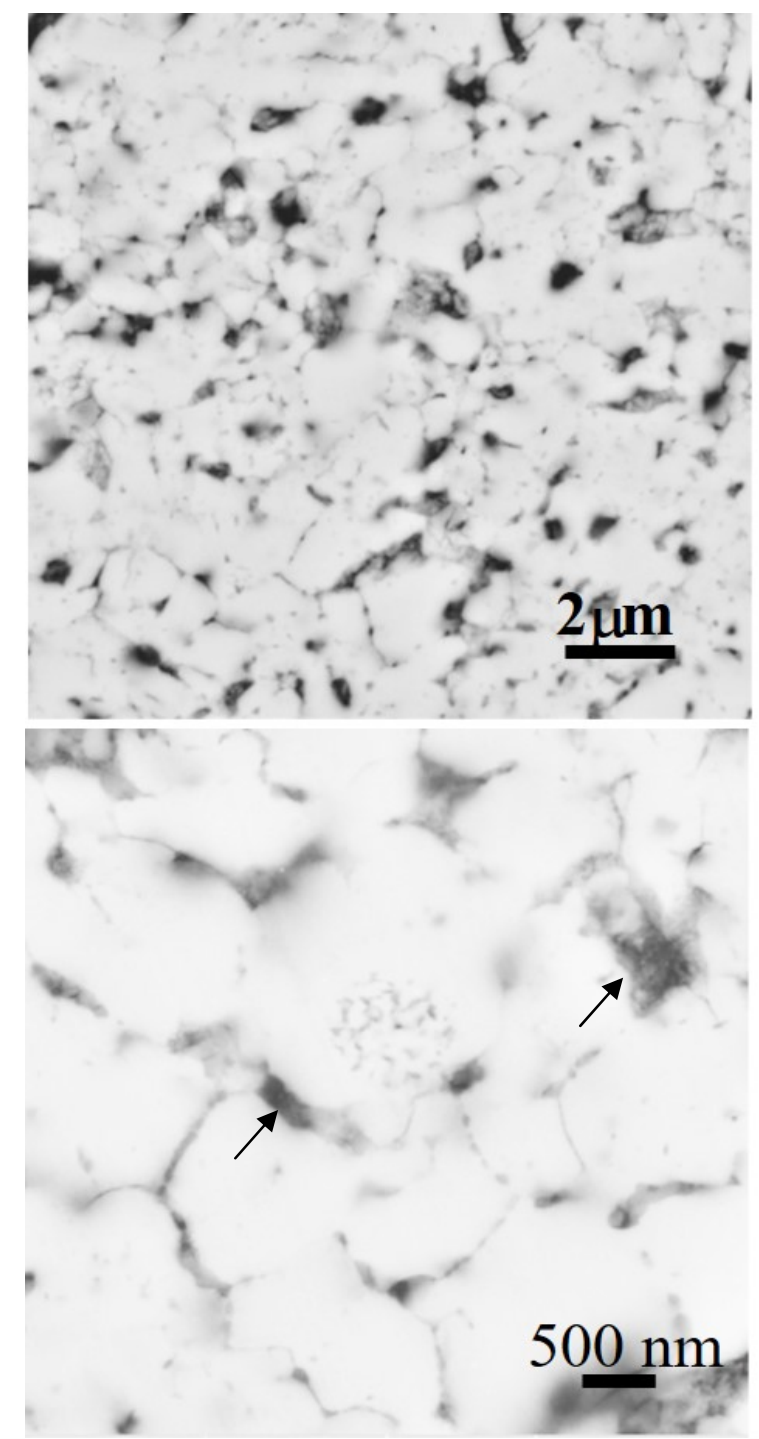

Fig. 7 The SEM images of FeSe samples produced by a sintering at $900{ }^{\circ} \mathrm{C}$ for 24 hours and ball milling time $6 \mathrm{~h}$.
We expect that further improvement in $J_{\mathrm{c}}$ in the bulk FeSe material can be realized by a further improving the grain boundary connection without impurities. The present results indicate that the optimization of high temperature sintering is crucial for improving the bulk FeSe performance, which can be very useful for the development of superconducting super-magnet applications.

\section{Summary}

In summary, we fabricated bulk polycrystalline $\mathrm{Ca}_{0.8} \mathrm{Fe}_{2} \mathrm{Se}_{2}$ via solid state reaction at different low and high temperatures. X-ray diffraction results clarified that at temperature above $350{ }^{\circ} \mathrm{C}$ the major phase was FeSe. Magnetization vs. temperature measurements showed a bump at temperatures between $133 \mathrm{~K}$ and $135 \mathrm{~K}$, which for very high sintering temperatures shifted downwards, up to $125 \mathrm{~K}$. Further, bulk FeSe pellets were produced utilizing ball-milled powders sintered at $900{ }^{\circ} \mathrm{C}$ for 24 hours. These samples showed a sharp superconducting transition at around $8.16 \mathrm{~K}$ and $J_{c}$ value of $6,253 \mathrm{~A} / \mathrm{cm}^{2}$ at $5 \mathrm{~K}$ in self field. The SEM analysis indicated a dense structure of grains of about $2 \mu \mathrm{m}$ in size, SEM by EDX analysis proved that the grains are mostly of FeSe. A further optimization of the sintering process is still needed to obtain good quality superconducting FeSe that could be utilized as a rare-earth-free superconducting 
super-magnet in industrial applications, e.g. in medicine.

\section{Acknowledgments}

The paper was supported by Grant-in-Aid FD research budget code: 112261, SIT (Shibaura Institute of Technology) and by APVV No. 0330-12, VEGA 2/0121/16.

\section{References}

[1] Kamihara, Y., Watanabe, T., Hirano, M., and Hirano, H. 2008. "Iron-based Layered Superconductor $\mathrm{La}\left[\mathrm{O}_{1-x} \mathrm{~F}_{x}\right] \mathrm{FeAs}(x=0.05-0.12)$ with $\mathrm{T}_{\mathrm{c}}=26 \mathrm{~K}$." J. Am. Chem. Soc. 130: 3296-7.

[2] Chen, X. F., Wu, T., Wu, G., Liu, R. H., Chen, H., and Fang, D. F. 2008. "Superconductivity at $43 \mathrm{~K}$ in $\mathrm{SmFeAsO}_{1-x} \mathrm{~F}_{x}$." Nature 453: 761-2.

[3] Yang, J., Li, Z. C., Lu, W., Yi, W., Shen, X. L., Ren, Z. A., Che, G. S., and Dong, X. L. 2008. "Superconductivity at $53.5 \mathrm{~K}$ in $\mathrm{GdFeAsO}_{1-x}$." Supercond. Sci. Technol. 21 (082001): 5 .

[4] Wu, M. K., Hsu, F. C., Yeh, K. W., Huang, T. W., Luo, J. Y., Wang, M. J., Chang, H. H., Chen, T. K., Rao, S. M., Mok, B. H., Chen, C. L., Huang, Y. L., Ke, C. T., Wu, P. M., Chang, A. M., Wu, C. T., and Perng, T. P. 2009. "The Development of the Superconducting PbO-type $\beta-F e S e$ and Related Compounts." Physica C 469: 340-9.

[5] Mizuguchi, Y., Tomioka, F., Tsuda, S., Yamaguchi, T., and Takano, Y. 2008. "Superconductivity at $27 \mathrm{~K}$ in Tetragonal FeSe under High Pressure." Appl. Phys. Lett. 93 (152505): 3.

[6] Mizuguchi, Y., Tomioka, F., Tsuda, S., Yamaguchi, T., and Takano, Y. 2009. "Superconductivity in S-Substituted FeTe." Appl. Phys. Lett. 94 (012503): 3.

[7] Yeh, K. W., Huang, T. W., Huang, Y. L., Chen, T. K., Hsu, F. C., and Wu, P. M. 2008. "Tellurium Substitution Effect on Superconductivity of the $\alpha$-phase Iron Selenide." Europhys Lett 84 (37002): 4.

[8] Guo, J., Jin, S., Wang, G., Wang, S., Zhu, K., Zhou, T., He, M., and Chen, X. 2010. "Superconductivity in the
Iron Selenide $\mathrm{K}_{x} \mathrm{Fe}_{2} \mathrm{Se}_{2}(0 \leq x \leq 1.0)$." Phys. Rev. $B 82$ (180520R): 4.

[9] Jaroszynski, J., Hunte, F., Balicas, L., Younjung Jo, Raicevic, I., Gurevich, A., Larbalestier, D. C., Balakirev, F. F., Fang, L., Cheng, P., Jia, Y., and Wev, H. H. 2008. "Upper Critical Fields and Thermally-Activated Transport of $\mathrm{NdFeAsO}_{0.7} \mathrm{~F}_{0.3}$ Single Crystals." Phys. Rev. B 78 (174523): 5.

[10] Senatore, C., Flukiger, R., Cantoni, M., Wu, G., Liu, R. H., and Chen, H. H. 2008. "Upper Critical Fields Well above $100 \mathrm{~T}$ for the Superconductor $\mathrm{SmFeAsO}_{0.85} \mathrm{~F}_{0.15}$ with $\mathrm{T}_{\mathrm{c}}=65$ K." Phys. Rev. B 78 (0545i4): 4.

[11] Ying, T. P., Chen, X. L., Wang, G., Jin, S. F., Zhou, T. T., Lai, X. F., Zhang, H., and Wang, W. Y. 2012. "Observation of Superconductivity at 30-46 K in $\mathrm{A}_{\mathrm{x}} \mathrm{Fe}_{2} \mathrm{Se}_{2}(\mathrm{~A}=\mathrm{Li}, \mathrm{Na}, \mathrm{Ba}, \mathrm{Sr}, \mathrm{Ca}, \mathrm{Yb}$, and Eu)." Science Reports 2 (426): 5.

[12] Janaki, J., Geetha, K. T., Mani, A., Kalavathi, S., and Reddy, GVR. 2009. "Synthesis, Characterization and Low Temperature Studies of Iron Chalcogenide Superconductors." J. Alloys Compd. 486: 37-41.

[13] Mizuguchi, Y., Tomioka, F., Tsuda, S., Yamaguchi, T., and Takano, Y. 2008. "Superconductivity at $27 \mathrm{~K}$ in Tetragonal FeSe under High Pressure." Appl. Phys. Lett. 93 (152505): 3.

[14] Li, X., Liu, Y., Ma, Z., and Gao, Z. 2013. "Characterization of Low-Temperature Synthesized $\mathrm{FeCr}_{0.05}$ Se Superconductors." Cryogenic (55-56): 68-72.

[15] Onar, K., and Yakinci, M. E. 2015. "Solid State Synthesis and Characterization of Bulk-FeSe Superconductors." Journal of Alloys and Compounds 620: 210-6.

[16] Yoo, J. S., Jong, B. H., Byeong, H. M., Yong, S. K., Kyu, J. L., Myung, H. J., and Jong-Soo, R. 2011. "Superconducting Properties of a Stoichiometric FeSe Compound and Two Anomalous Features in Normal State." Journal of the Korean Physical Society 59: 312-6.

[17] Ozki, T., Deguchi, K., Mizuguchi, Y., Kawasaki, Y., Tanaka, T., Yamaguchi, T., Kumakura, H., and Takano, Y. 2012. "Fabrication of Binary FeSe Superconducting Wires by Diffusion Process." J. of Apply. Phys. 111 (112620): 4. 